\title{
ON CANONICAL FORMS
}

\author{
By E. K. WAKEFORD.
}

[Read February 12th, 1920.]

[THIs paper consists of the second part of a dissertation "General Theorems and Canonical Forms," written by Mr. Wakeford in the spring of 1916. As it was almost complete in itself it has been thought best to print it separately, with a few words of explanation to enable the reader to grasp the principler on which the argument rests.

Suppose it is required to show that a general ternary cubic in $x, y, z$ can, by a linear transformation, be reduced to the form

$$
X^{8}+Y^{3}+Z^{3}+6 m X Y Z
$$

then we have to identify the given cubic with

$$
\begin{aligned}
\left(\alpha_{1} x+a_{2} y+a_{3} z\right)^{3} & +\left(\beta_{1} x+\beta_{3} y+\beta_{3} z\right)^{3}+\left(\gamma_{1} x+\gamma_{2} y+\gamma_{3} z\right)^{3} \\
& +6 m\left(a_{1} x+\alpha_{2} y+\alpha_{3} z\right)\left(\beta_{1} x+\beta_{2} y+\beta_{3} z\right)\left(\gamma_{1} x+\gamma_{2} y+\gamma_{3} z\right),
\end{aligned}
$$

i.e. we have to show that the last expression is capable of representing the general cubic in $x, y, z$.

$$
\text { Writing it as } \quad \Sigma a_{\mu^{\prime \prime}} \cdot x^{p} y^{\prime \prime} z^{\prime},
$$

it suffices to prove that the $a$ 's are independent functions of the ten variables ronsisting of the $a$ 's, $\beta$ 's, $\gamma$ 's and $m$.

This amounts to proving that the Jacobian does not vanish identically, but it will be seen $(\$ 2)$ that $\mathrm{Mr}$. Wakeford does not use the Jacobian explicitly. He rather assumes the existence of a relation between the $a$ 's, and makes a deduction therefrom which is easily turned into a test to determine whether a proposed canonical form is possible or not.*

* The idea of using the Jacobian seems to be due to Kronecker: cf. Lasker, Math. Annalen, Vol. 58 (1904), pp. 434-446. The methods there used are the same as Mr. Wakeford's in principle, but the form is not so convenient." Several references being mude to Elliott (Algebra of Quantics) and Richmond ("On Canonical Forms," Quarterly Journal of Math., Vol. 33 (1902), pp. 331-310), they are quoted as "Quantics" and "Richmond" respectively. 
The writer's thesis is to establish the possibility or otherwise of a given reduction not to find the reducing process or the number of solutions.-J. H. G.]

1. The problem of reducing a $q$-ary $p$-ic, that is to say a quantic of order $p$ in $q$ variables. to a canonical form, consists of expressing the quantic

$$
\sum_{s=1}^{m} \alpha_{s} x_{1}^{s p} x_{2}^{s^{p}} \ldots x_{q}^{s^{\prime}}
$$

in the proposed form

$$
F \equiv \sum_{s=1}^{m} x_{1}^{p_{1}} x_{2}^{p_{2}} \ldots x_{q_{1}}^{s p_{q}} f_{s}\left(l_{1}, l_{2}, \ldots, l_{n}\right),
$$

where $l_{1}, l_{2}, \ldots, l_{n}$ are (usually) independent variables, and

$$
{ }_{s} p_{1}+{ }_{s} p_{2}+\ldots+{ }_{s} p_{q}=p \quad(s=1,2, \ldots, m) .
$$

It will be noticed that $F$ appears to differ from some proposed forms in that no indefinite linear forms occur in it. If such a form $X$ should occur, write

$$
X \equiv x_{1} \lambda_{1}+x_{2} \lambda_{2}+\ldots+x_{q} \lambda_{q}
$$

and the coefficients $\lambda$ take their place among the variables $l$. Thus any number of indefinite quantics of linear or higher order may be disposed of, and $F$ obtained in the form above.

2. The proposed form $F$ is or is not canonical according as the functions $f_{1}, f_{2}, \ldots, f_{m}$ are or are not independent.

Suppose that the form is not canonical, so that a relation $\psi(f)=0$ exists. Then

$$
\frac{\partial f_{1}}{\partial l_{r}} \frac{\partial \psi}{\partial f_{1}}+\frac{\partial f_{2}}{\partial l_{r}} \frac{\partial \psi}{\partial f_{2}}+\ldots+\frac{\partial f_{m}}{\partial l_{r}} \frac{\partial \psi}{\partial f_{m}}=0 \quad(r=1,2, \ldots, n) .
$$

Now

$$
\frac{\partial F}{\partial l_{r}} \equiv \sum_{s=1}^{m} \frac{\partial f_{2}}{\partial l_{r}} x_{1}^{s} p_{1} x_{2}^{s} p_{2} \ldots x_{q}^{s} p_{4}
$$

The contragredient quantic $\Phi$ defined by

$$
\Phi \equiv \sum_{s=1}^{m} \frac{\partial \psi}{\partial f_{1}} u_{1}^{s} p u_{2}^{s p_{q}} \ldots u_{q}^{s} p_{q} \frac{p !}{{ }_{s} p_{1} !{ }_{s} p_{2} ! \ldots p_{q} !}
$$


where $u_{1}, u_{2}, \ldots, u_{q}$ correspond with $x_{1}, x_{2}, \ldots, x_{q}$ respectively, is apolar to $\partial F / \partial l_{r}$, in virtue of relation (1). Hence if $F$ is not canonical the quantics $\partial F / \partial l_{r}$ are all apolar to a certain contragredient quantic. Note that this apolar form must exist for all values of $l$. [If $\partial \psi / \partial f_{\text {s }}$ $(s=1,2, \ldots, m)$ vanishes for a certain set of values of $l_{1}, \ldots, l_{n}$, two apolar forms instead of one are obtained.] In order therefore to prove that $F$ is canonical, it is only necessary to find a particular set of values of $l_{1}, l_{2}, \ldots, l_{n}$, so that the quantics $\partial F / \partial l_{r}$ have no form apolar to them.

Again, if such an apolar form $\Phi$ exists for general values of $l$, the relations following hold good for suitable values of $\lambda$,

$$
\begin{aligned}
& \frac{\partial f_{1}}{\partial l_{1}} \lambda_{1}+\frac{\partial f_{2}}{\partial l_{1}} \lambda_{2}+\ldots+\frac{\partial f_{m n}}{\partial l_{1}} \lambda_{m}=0, \\
& \frac{\partial f_{1}}{\partial l_{2}} \lambda_{1}+\frac{\partial f_{2}}{\partial l_{2}} \lambda_{2}+\ldots+\frac{\partial f_{n}}{\partial l_{2}} \lambda_{m}=0, \\
& \ldots \quad \ldots \quad \ldots \quad \ldots \quad \ldots \\
& \ldots \quad \ldots \quad \ldots \quad \ldots+\frac{\partial f_{m}}{\partial l_{n}} \lambda_{m}=0 .
\end{aligned}
$$

The Jacobian of the $m$ functions with respect to any $m$ of the $n$ variables $l$ must therefore vanisl. in general, and so vanish always. Hence the functions are not independent, and the form is not canonical. If there is no apolar form, then

$$
\lambda_{1} \frac{\partial F}{\partial l_{1}}+\lambda_{2} \frac{\partial F}{\partial l_{2}}+\ldots+\lambda_{n} \frac{\partial F}{\partial l_{n}}
$$

can be made to represent any $q$-ary $p$-ic for suitable values of $\lambda_{1}, \lambda_{2}, \ldots, \lambda_{n}$, and conversely.

3. It has hitherto been required that all implicit parameters in $F$, such as those contained in a linear form $X$, should be written out explicitly: e.g.

$$
X \equiv \lambda_{1} x_{1}+\lambda_{2} x_{2}+\ldots+\lambda_{q} x_{1 .}
$$

This may be avoided, for if

$$
\frac{\partial F}{\partial X_{r}} \equiv x_{r} \frac{\partial F}{\partial X} \quad(r=1,2, \ldots, q)
$$

is apolar to $\Phi$, the quantic $\partial F / \partial X$ of order $p-1$ is apolar to $\Phi$ : and conversely if $\partial F / \partial X$ is apolar to $\Phi$, then $x_{r} . \partial F / \partial X$ is also apolar to $\Phi$. 
Thus suppose the quantic is the binary quartic, and

$$
F \equiv X^{4}+6 m X^{2} Y^{2}+Y^{4}
$$

Then

$$
\begin{aligned}
& \frac{\partial F}{\partial X} \equiv X^{3}+3 m X Y^{2}, \\
& \frac{1}{4} \frac{\partial F}{\partial Y} \equiv 3 m X^{2} Y+Y^{3},
\end{aligned}
$$

and

$$
\frac{1}{6} \frac{\partial F}{\partial m} \equiv X^{2} Y^{2}
$$

are apolar to $\Phi$. Put $m=0$, and it is clear that $\Phi$ does not exist, and the form is canonical, since no binary quartic has $X^{3}, Y^{3}$, and $X^{2} Y^{2}$ apolar to it.

A similar method may be adopted in case an indefinite quantic of the second or higher order occurs in $F$. Thus in order to justify

$$
F \equiv c_{2} c_{3}-c_{1}^{2},
$$

for the ternary quartic, where $c_{1}, c_{2}, c_{3}$ are conics, it is only necessary to consider that

$$
\begin{aligned}
-\frac{\partial}{2} \frac{\partial F}{\partial c_{1}} & \equiv c_{1}, \\
\frac{\partial F}{\partial c_{2}} & \equiv c_{3}, \\
\frac{\partial F}{\partial c_{3}} & \equiv c_{2} ;
\end{aligned}
$$

and there is not always a class quartic to which those conics are apolar, as may be seen by considering the case

$$
o_{1} \equiv x_{1}^{2}, \quad c_{2} \equiv x_{2}^{2}, \quad c_{3} \equiv x_{3}^{2} .
$$

Now take the case when the proposed form is the sum of $n$ perfect $p$-th powers of linear form, that is to say

$$
F \equiv X_{1}^{p}+X_{2}^{p}+\ldots+X_{n}^{p}
$$

If $\Phi$ exists $X_{r}^{n-1}(r=1,2, \ldots, n)$ is apolar to it. It will now be convenient to reciprocate the problem. Suppose $S$ is a quantic of order $p$ in 
$x_{1}, x_{2}, \ldots, x_{t}$, and $U^{p-1}$ is apolar to $S$. Considering $S$ as denoting a hyper-surface, and $U$ a point, it follows that the $(p-1)$-th polar of the hyper-surface with respect to the point vanishes, so that the point is a double point on the hyper-surface. Hence, if in the original problem $\Phi$ exists, there must be a hyper-surface of order $p$ having $n$ arbitrary double points. If for one set of points there is no such hyper-surface, $F$ is canonical. If, on the other hand, a method of finding such a surface for the general case can be given, $F$ is not canonical. A number of well known results follow immediately from this geometrical aspect of the problem, which often enables a solution to be obtained without putting pen to paper.

(1) The binary $(2 n-1)$-ic can be expressed as the sum of $n$ form $X_{r}^{2 n-1}$, for no $(2 n-1)$-ic can have double points at $n$ different points.

(2) The ternary quadratic cannot be expressed as the sum of two squares, for the square of the line joining two points is a conic with double points at both of them.

(3) The ternary quartic cannot be expressed as the sum of five fourth powers, for the square of the conic through five points is a quartic with double points at all of them. [Quantics, $\$ 230 ;$ Richmond, \$9.]

(4) The ternary quintic can be expressed as the sum of seven fifth powers, for if no six of seven points lie on a conic, it is easy to show that no quintic can have nodes at all of them. [Richmond, $\$ 10$.

(5) The quaternary cubic can be expressed as the sum of five cubes, for no cubic surface can have five double points of which four do not lie on a plane. [Consider twisted cubics through the five points; Richmond, $\$ 11$.

(6) The quinary cubic cannot be expressed as the sum of seven cubes, for through seven points in four dimensions passes a quartic curve, the chords of which generate a cubic hyper-surface containing the curve as a double curve. [Richmond, $\$ 12$.

4. The binary $2 n-i c$,

$$
F \equiv X_{1}^{2 n}+X_{2}^{2 n}+\ldots+X_{n}^{2 n}+\frac{2 n !}{n !} m X_{1}^{2} X_{2}^{2} \ldots X_{n}^{2} .
$$


In this case $\Phi$, if it exists, has

and

$$
\begin{gathered}
\frac{1}{2 n} \frac{\partial F}{\partial X_{r}} \equiv X_{1}^{2 n-1}+\frac{(2 n-1) !}{2^{n-1}} m X_{1}^{\vdots} \ldots X_{r-1}^{2} X_{r} X_{r+1}^{2} \ldots X_{n}^{2}, \\
\frac{\partial F}{\partial m} \equiv \frac{2 n !}{2^{n}} X_{1}^{2} X_{2}^{2} \ldots X_{n}^{\prime \prime}
\end{gathered}
$$

apolar to it. Put $m=0$. Then $\Phi$ is a $2 n$-ic with $X_{1}, X_{2}, \ldots, X_{n}$ as double points, that is $\Phi$ is $X_{1}^{2} X_{2}^{2} \ldots X_{n}^{2}$. But this form is not always apolar to itself, unless $n=1$; hence the proposed form $F$ is canonical for all binary $2 n$-ics except the quadratic.

The number of ways in which this reduction can be performed is interesting. For $n=2,3,4$, the numbers are $3,8,5$ respectively. [Quantics, $\$ 211$; Wakeford, "A Canonical Form of the Binary Sextic," Messenger of Mathematics, Vol. 43 (1914), pp. 25-28; and Quantics, $\$ 227$.

It is clear that instead of $X_{1}^{2} X_{2}^{2} \ldots X_{n}^{2}$, any function of $X_{1}, X_{2}, \ldots, X_{n}$ which is not alwilys apolar to $X_{1}^{2} X_{2}^{2} \ldots X_{n}^{2}$ may be taken. [Quantics, $\$ 225$.]

5. Any quantic of order $p>1$ may have its terms of the form $x_{1}^{p-1} x_{2}$ removed. Let $F$ be the general $p$-ic in $q$ variables $X_{1}, X_{2}, \ldots, X_{n}$, without such terms as $X_{1}^{p-1} X_{2}$.

Consider any term of $F$, e.g. $k X_{1}^{p-2} X_{2} X_{3} . \quad \Phi$ if it exists will be apolar to $\partial F / \partial k$, i.e. $X_{1}^{p-2} X_{2} X_{3}$. Now $\Phi$ may be written in terms of $U_{1}, U_{2}, \ldots, U_{l}$, where $U_{1}$ is the common point of the linear forms $X_{2}, X_{3}, \ldots, X_{q}$. Written thus, $\Phi$ evidently does not contain the term $U_{1}^{p-2} U_{2} U_{3}$. Similarly for all the other terms of $F$. Hence $\Phi$ consists entirely of such terms as $U_{1}^{p-1} U_{2}$.

Consider $\partial F / \partial X_{1}$, which is apolar to $\Phi$. Put the coefficients of all the terms of $F$ zero, except the terms $X_{r}^{p}$. Then $\partial F / \partial X_{1}$ is $k X_{1}^{p-1}$ So $\Phi$ can contain no coefficient of $U_{1}^{p-1}$, or similarly of $U_{r}^{p-1}$ Hence $\Phi$ does not exist, since it has been shown to contain no terms except those of the form $U_{1}^{p-1} U_{2}$. Hence the proposed form is canonical. Particular cases are canonical forms of the binary cubic, quartic, and quintic, and of the ternary cubic.

A similar proof shows that all terms of the form $x_{1}^{p-r} x_{2}^{r}$ can be removed, where $r$ is any fixed number. The following question now arises:-Is 
there any ready test by which, given $q(q-2)$ terms of a quantic, it can be decided whether they are removable or not?

It is easy to prove by the method above that the following test is necessary :-It must be possible to associate with each of the terms to be removed a different one of the ratios

$$
x_{i} / x_{*} \quad(r=1,2, \ldots, q ; s=1,2, \ldots, q ; r \neq s),
$$

so that the product in each case is a term which is not to be removed.

I cannot prove this condition sufficient, though it seems to be so.

The following particular form is certainly sufficient:-Choose any set of terms which are prime to one another, that is to say such that the same $x_{r}$ cannot occur in two of them. These terms may be "isolated" (except in the case of linear forms), i.e. all terms which can be found by multi. plying them by $x_{r} / x_{s}$ may be removed.

For instance, $x_{1}^{p}, x_{2}^{p}, \ldots, x_{n}^{\nu}$ are isolated if the terms $x_{r}^{r-1} x_{s}$ are removed.

6. The lines on a cubic surface.-It may be inadvisable to make explicit use of the apolar form $\Phi$. It order to dispense with it, note that since the general $q$-ary $p$-ic contains $m$ terms, and the forms $\partial F / \partial l_{r}$ are all apolar to $\Phi$, a syzygy must exist between any $m$ of the $n$ forms $\partial F / \partial l_{r}$ Conversely, if that is true, $\Phi$ must exist. The simplest case is where $n=m$, then the form is canonical or not according as there does or does not exist a syzygy between the forms $\partial F / \partial l_{r}$. In dealing with a linear form $X$, the term in the syzygy corresponding to $\partial F / \partial X$ is $X^{\prime} . \partial F / \partial X$, where $X^{\prime}$ is an arbitrary linear form. The following example is written out at length.

The study of the lines on a cubic surface may be started by taking

$$
F \equiv X_{1} X_{2} X_{3}-X_{4} X_{5} X_{6}
$$

as the canonical form of a cubic surface. In order to justify this, write

$$
X_{r} \equiv l_{r} x_{1}+m_{r} x_{2}+n_{r} x_{3}+p_{r} x_{4} \quad\left(r=1,2, \ldots, 6 ; p_{r} \neq 0\right) .
$$

Consider the twenty cubic surfaces

$$
\begin{array}{lllllll}
X_{1} X_{2} X_{3}, & X_{2} X_{3} x_{1}, & X_{2} X_{3} x_{2}, & X_{2} X_{3} x_{3}, & X_{3} X_{1} x_{1}, & X_{3} X_{1} x_{2}, & X_{3} X_{1} x_{9}, \\
X_{1} X_{2} x_{1}, & X_{1} X_{2} x_{2}, & X_{1} X_{2} x_{8}, & X_{4} X_{5} X_{6}, & X_{5} X_{6} x_{4}, & X_{5} X_{6} x_{5}, & X_{5} X_{6} x_{6}, \\
X_{6} X_{4} x_{1}, & X_{6} X_{4} x_{2}, & X_{6} X_{4} x_{3}, & X_{4} X_{5} x_{1}, & X_{4} X_{5} x_{2}, & X_{4} X_{5} x_{9} .
\end{array}
$$


These surfaces are all apolar to $\Phi$, if it exists. Hence, since there are only twenty terms in the equation of a cubic surface, a syzygy must connect the surfaces, viz.

$$
X_{2} X_{9} X_{1}^{\prime}+X_{3} X_{1} X_{2}^{\prime}+X_{1} X_{2} X_{3}^{\prime} \equiv X_{5} X_{6} X_{4}^{\prime}+X_{6} X_{4} X_{5}^{\prime}+X_{4} X_{4} X_{(,}^{\prime},
$$

where neither side can vanish, since $p_{r} \neq 0$.

Consider the cubic surface represented by both sides of this syzygy. It contrins the six lines in which $X_{2} X_{3}, X_{3} X_{1}, X_{1} X_{2}, X_{5} X_{6}, X_{6} X_{4}$, $X_{4} X_{5}$ respectively intersect. The plane $X_{1}$ meets the surface in two lines, viz. $X_{1} X_{2}, X_{1} X_{3}$. The three lines $X_{5} X_{6}, X_{6} X_{4}, X_{4} X_{5}$ accordingly meet $X_{1}$ in collinear points (or else on one of these two lines), and this is not the case for all sets of six planes $X_{r}$. Hence the syzygy is impossible and the form canonical. 